Meta

Journal des traducteurs

Translators' Journal

\title{
Journées de traduction, stages internes, stages externes
}

\section{Daniel Gouadec}

Volume 24, numéro 4, décembre 1979

URI : https://id.erudit.org/iderudit/002950ar

DOI : https://doi.org/10.7202/002950ar

Aller au sommaire du numéro

Éditeur(s)

Les Presses de l'Université de Montréal

ISSN

0026-0452 (imprimé)

1492-1421 (numérique)

Découvrir la revue

Citer cet article

Gouadec, D. (1979). Journées de traduction, stages internes, stages externes. Meta, 24(4), 423-433. https://doi.org/10.7202/002950ar d'utilisation que vous pouvez consulter en ligne.

https://apropos.erudit.org/fr/usagers/politique-dutilisation/ 


\title{
Journées de traduction, stages internes, stages externes
}

\author{
Daniel Gouadec
}

\begin{abstract}
Dans la masse de commentaires suscités par les programmes universitaires de formation de traducteurs, il est sans aucun doute utile de retenir deux avis convergents. Les étudiant(e)s reprochent trop souvent aux écoles de «ne pas leur avoir appris ce qu'était véritablement le métier de traducteur » et les employeurs affirment avec force que la formation universitaire n'est pas \& adaptée aux besoins réels de la profession et de l'entreprise $\$$. Il est futile de rouvrir ce vieux débat si l'on ne réfléchit pas aux moyens propres à accroître le rendement de la formation universitaire. Parmi ces moyens, le présent article traitera des journées de traduction et stages en école, tirant les leçons d'une expérience réussie - aux dires des participants - avant de considérer les modalités permettant une généralisation et un développement des activités pratiques de traduction en école.
\end{abstract}

\section{L'EXPÉRIENCE}

Entreprise à l'Université d'Ottawa au cours de l'année universitaire 19781979 , l'expérience avait pour but, par l'organisation de journées de traduction, de placer les étudiants dans des conditions de travail aussi proches que possible des conditions réelles que rencontre le traducteur professionnel. Elle a touché un groupe de douze étudiants de troisième année d'Université (seconde année en traduction) inscrits à un cours de traduction générale. Les journées de traduction relevaient de ce cours dont elles constituaient le noyau de travaux pratiques. Trois journées de traduction se sont déroulées selon les modalités décrites ci-dessous.

Première journée de traduction (janvier) :

Remise d'un texte de 1400 mots aux participants le mercredi soir. La journée de traduction proprement dite se déroulant le vendredi suivant, les étudiants ont disposé de 24 heures (moins le temps consacré aux cours du jeudi) pour effectuer la recherche documentaire et terminologique. Le vendredi a été consacré à la traduction du texte dont une version manuscrite devait être remise huit heures après le début du travail. Cette version manuscrite a servi de base de comparaison avec une seconde version, dactylographiée cette fois, remise le lundi matin suivant. Chacun des participants pouvait remanier et modifier sa traduction à loisir durant la fin de semaine. 
Cette première « journée » s'est étalée sur trois jours parce que :

- l'inquiétude manifestée par les étudiants avant le premier contact avec ce type d'exercice justifiait une certaine souplesse dans l'organisation du travail ;

- il semblait surtout souhaitable de disposer, avant que ne commence la traduction proprement dite, des résultats des recherches et de corriger certaines erreurs;

- le fait que les recherches aient été effectuées avant que ne commence la traduction avait une intention pédagogique : il s'agissait d'obliger les participants à découper l'exercice en phases successives en évitant qu'une pré-traduction ne vienne fausser la démarche; il s'agissait aussi de se placer dans une situation d'observation des seuls problèmes de transfert ;

- l'intervalle entre la fin de la traduction (version manuscrite) et la remise du texte définitif (version dactylographiée) a été introduit de manière à permettre aux étudiants de revoir leur production d'un œil neuf. Les écarts importants entre les deux versions ont prouvé la validité d'une révision personnelle à froid. Ces mêmes écarts étaient riches d'enseignements pour le responsable du cours dans la mesure où ils témoignaient de démarches nettement caractérisées induisant soit des améliorations sensibles, soit des dégradations catastrophiques mais extrêmement révélatrices.

Seconde et troisième journées de traduction (février, mars) :

$\mathrm{Au}$ cours de ces deux journées, afin de réduire l'écart entre les conditions professionnelles et pédagogiques, toutes les activités ont été bloquées sur un seul jour. Les textes à traduire ( 1100 puis 1300 mots) ont été remis le matin même, et les participants sont demeurés libres d'organiser comme ils l'entendaient les diverses phases de leur travail. La seule condition imposée était la remise obligatoire d'une version définitive dactylographiée au plus tard huit heures après la remise des textes à traduire. Il est entendu que, dans tous les cas, les participants pouvaient, s'ils le désiraient, recourir à tous les moyens de documentation et à toutes les sources de renseignements imaginables.

Les traductions remises par les étudiants ont, chaque fois, été transmises au Bureau des traductions ${ }^{1}$ où elles ont fait l'objet d'une révision de type «professionnel \%. Une fois les révisions effectuées, les réviseurs concernés ${ }^{2}$ sont venus, pendant une après-midi entière, commenter devant les participants leurs révisions. Ces rencontres ont constitué l'un des aspects les plus positifs de l'expérience.

BILAN

La mise en place de journées de traductions suivies de demi-journées de révision commentée ne va pas sans problèmes. Il serait vain de se voiler la face

1. Nous tenons à exprimer nos remerciements à $\mathbf{M}$. Boisvert, directeur de la Division de la formation au Bureau des traductions à Ottawa. Sans sa précieuse collaboration, ces journées n'auraient pu se dérouler selon les modalités prévues.

2. Nous tenons également à remercier Mlle Goffart et M. Godbout, de la Division de la formation au Bureau des traductions à Ottawa, qui ont bien voulu fournir les textes à traduire, réviser les traductions des étudiants ef consacrer, à chaque fois, une demijournée aux bilans et commentaires. Sans leur aide, l'expérience n'aurait sans doute porté que des fruits secs. 
et de dire que ces problèmes peuvent, toujours et partout, se trouver aisément résolus.

Tout d'abord se pose le problème de la révision « externe» - à supposer que l'on ait recours à ce type de révision qui, d'après les étudiants concernés, est nécessaire ou indispensable. Il s'avère que, dans ce cas, la collaboration dévouée de réviseurs professionnels et de leurs employeurs soit une condition sine qua non.

Ensuite se pose le problème de la taille des groupes d'étudiants. Il est difficile d'obtenir de bons résultats si le groupe dépasse quinze participants. Ceci ne constitue un handicap que dans certaines sections de seconde année. Encore serait-il possible, pour certains groupes, d'échelonner les journées de traduction en constituant des sous-groupes qui, à tour de rôle, connaîtraient les épuisantes journées de traduction. L'objection « naturelle» serait que cette multiplication des journées pour un enseignant donné représente une masse de corrections et une série importante de présences sur le campus. Mais les journées de correction peuvent remplacer avantageusement les travaux pratiques.

Restent les contraintes imposées par les emplois du temps et la collaboration indispensable entre enseignants responsables des divers cours touchant aux activités de traduction. Ces contraintes ne devraient pas s'avérer déterminantes en regard des multiples avantages que les uns et les autres peuvent retirer des « journées ».

Parmi les avantages, on notera d'abord les avantages évidents ou «transparents »:

- les conditions de travail s'apparentent aux conditions réelles que connaît tout professionnel ;

- le volume de traduction effective contrôlée augmente pour chacun des étudiants concernés ${ }^{3}$;

- les recherches documentaires et terminologiques deviennent rentables. Elles deviennent en même temps impératives. La rentabilité est évidente : il est clair que, dans la traduction de plus de mille mots, tout élément de recherche sert plus souvent que dans la traduction de deux cents ou trois cents mots. La nécessité, par ailleurs, dérive du fait que l'apprenti-traducteur ne veut pas perdre la face devant un réviseur autre que son professeur ;

- les participants sont obligés d'organiser leur travail afin de parvenir à une traduction définitive avant la fin de la journée. Il ne s'agit plus de se dire qu'on a le temps ou que l'on reprendra tout cela demain ;

- chaque étudiant peut juger, au terme d'une journée de traduction organisée selon les modalités décrites, si le type de contraintes imposé par l'exercice est

3. Il est intéressant de noter que les étudiants les plus faibles du groupe ayant subi le programme des journées de traduction ont, à l'occasion d'une épreuve en temps limité servant de contrôle ultime, traduit un texte de 200 mots avec une rapidité (qui n'a pas nui à la qualité) dépassant la vitesse normale de travail d'étudiants de même niveau. Il semblerait donc que la pression subie pendant les journées de traduction contribue à accroître le rendement quantitatif. 
surmontable ou si, au contraire, il ou elle préférerait connaître, dans une autre profession, d'autres types de contraintes ou pas de contraintes du tout.

Parmi les avantages moins évidents mais autrement significatifs, on notera :

- l'occasion offerte aux enseignants responsables des cours de traduction ou de documentation ou de terminologie de suivre l'étudiant dans la progression de sa démarche. Ils peuvent « voir» comment chacun organise sa documentation, ses recherches, ses consultations. Ils peuvent juger directement des résultats, suivre le cheminement de la pensée (et de la parole, car on discute beaucoup en journée de traduction). Ils peuvent analyser et juger les stratégies adoptées par chacun des participants et ils peuvent surtout déterminer, avec une rigueur qui ne permet aucun autre type d'exercice, quelles sont les difficultés, quels sont les problèmes réels de chacun dans une situation réelle de traduction. La situation est alors totalcment différente de celle que l'on rencontre dans le schéma habituel du cours suivi de travaux pratiques puis de correction des travaux pratiques. Devant une copie d'étudiant, on ne peut que supposer que telle ou telle erreur ou faute intervient pour telle ou telle raison. Devant l'étudiant au travail, on sait pourquoi il se produit des erreurs. Le diagnostic devient alors d'une précision absolue et l'application du remède immédiate ;

- la possibilité de définir, en situation de traduction et non dans le cadre désincarné du cours, des stratégies directement applicables puis de juger si ces stratégies sont appliquées avec succès ou efficacité. Il ne s'agit nullement de proposer des solutions aux problèmes rencontrés par l'étudiant mais de lui indiquer l'une des voies à suivre pour parvenir lui-même à ces solutions. Que l'enseignant trouve des solutions est une chose. Que l'étudiant en trouve par l'application d'une méthodologie en est une autre. Encore faut-il qu'il soit guidé dans l'application d'une méthode;

- la possibilité de vérifier si les principes exposés dans tous les cours concernés sont a) utiles et b) appliqués ;

- la possibilité pour l'étudiant de s'initier au travail de groupe : les participants travaillent en collègues et non en concurrents ;

- la possibilité pour l'enseignant d'entreprendre des tests portant sur son groupe d'étudiants : tests de compréhension des textes, tests d'interprétation des textes, tests de mémorisation en relation avec la qualité des traductions produites, tests d'efficacité ;

- la possibilité d'exploiter des textes qui ne contiennent pas seulement tel ou tel type de difficulté ou dont le choix ne corresponde pas aux «dadas» de l'enseignant. Il est en effet impossible de trouver un texte de plus de mille mots qui, de ce point de vue, soit homogène.

La liste des avantages que présente ce type de travaux pratiques pourrait s'allonger encore, mais il faut sans doute laisser le soin à chacun de dresser son propre bilan. Nous souhaiterions néanmoins insister sur le fait que l'exercice offre l'occasion d'une sorte de «tradioscopie» dans la mesure où, pendant huit 
heures, on peut analyser les cheminements mentaux (et physiques) d'apprentistraducteurs sur lesquels s'exerce une pression certaine 4 .

Il faudrait aussi se garder d'oublier les avantages présentés par la révision externe. L'étudiant ne se heurte plus à la «sensibilité » de son professeur - un professeur qu'il connaît bien et dont il connaît surtout les préférences - mais aux exigences d'un réviseur qui, puisqu'il porte l'étiquette de professionnel, n'a pas à justifier ses exigences et n'a pas non plus à tenir compte en même temps de priorités ou d'options pédagogiques. Encore ne faudrait-il pas se méprendre sur le sens de ce terme : il y a sans nul doute une forme de thérapeutique pédagogique dans la révision externe. Il est à ce titre intéressant de noter que les étudiants concernés ont été frappés par le caractère "brutal » des révisions qu'ils ont jugées "franches et massives ». Mais, comme ils le disaient par ailleurs : «ça fait du bien, même si ça fait mal. »

Ce qui a marqué les spectateurs a été l'état d'énervement et parfois d'épuisement des participants aux journées de traduction. Cependant, si l'on en juge par le fait qu'ils ont réclamé la généralisation de ce type d'activités pratiques, on peut conclure que, malgré leur brutalité, ces expériences ont remporté un succès franc et peut-être massif.

\section{LES PROLONGEMENTS DE L'EXPÉRIENCE}

Devant les résultats de l'expérience entreprise, nous pensons qu'elle mérite d'être étendue. Il faudrait pour aela que la formation des traducteurs dans le cadre universitaire s'organise autour d'une structure de stages organisés sur une période recouvrant les trois années de formation universitaire prévues dans la plupart des programmes. Cette structure comporterait trois phases complémentaires, commencerait avec les journées de traduction, comporterait ensuite les stages « internes» effectués dans l'école puis serait couronnée par les stages 《 externes » effectués en dehors de l'école mais en cours d'année universitaire.

Phase un : journées de traduction - première année en école de traduction.

$\mathrm{Au}$ cours de la première année en école, let compte tenu du fait que les étudiants souhaitent traduire aussi vite que possible, il faudrait prévoir trois journées de traduction réparties sur le second semestre. Le premier trimestre serait réservé à l'approche méthodologique.

4. Parmi les résultats de ce type d'analyse, il en est un en particulier qui mérite réflexion : si l'on analyse les traductions successives d'un même passage présentant une difficulté pour un étudiant donné, on constate un phénomène d'érosion progressive de la partie comprise et traduite correctement d'emblée. L'étudiant tend à faire glisser insensiblement la portion traitée jusqu'à ce qu'une solution à la difficulté devienne acceptable. L'inconvénient est que cette démarche n'a pas pour effet de résoudre une difficulté en s'aidant de ce qui est bien compris et traduit mais d'imposer au texte une hypothèse - généralement erronée - concernant la partie délicate en faussant progressivement la partie traduite jusqu'à ce que le glissement de sens sur cette partie fasse que l'hypothèse paraisse acceptable. En d'autres termes, il n'impose pas au \& point noir " un cadre d'interprétation mais impose au contraire le «point noir » au cadre d'interprétation. 
Ces journées de traduction correspondraient au type présenté ci-dessus. Elles devraient, si possible, faire intervenir une révision externe ${ }^{5}$. Il existerait cependant une différence entre l'organisation de ces journées let le prototype de l'expérience décrite précédemment :

- les textes ne devraient pas dépasser une longueur de 600 à 800 mots ;

— la première journée serait une « journée triple » avec :

- une journée de préparation, recherche, documentation, dont l'organisation serait assurée par les professeurs de documentation et terminologie ;

- une journée de traduction dont l'organisation serait confiée au professeur de traduction;

- une journée de révision individuelle, de mise au point et de préparation matérielle de la version définitive.

— la seconde journée serait une «journée double» avec:

- une journée pour documentation et pré-traduction ;

- une journée pour re-traduction, révision, dactylographie.

- la troisième journée serait une «journée simple» avec remise du texte à traduire le matin et remise de la traduction le soir même.

Ces trois journées auraient pour objectif de permettre aux étudiants de se familiariser progressivement avec les contraintes de la traduction. Elles permettraient aux enseignants d'atteindre des objectifs précis : répertoire des difficultés, répertoire des erreurs de stratégie, répertoire des solutions à proposer, profil individuel de l'étudiant, adaptation des cours aux carences relevées, encadrement étroit des apprentis-traducteurs en cours d'exercice, etc.

Phase deux : stages internes - seconde année en école de traduction.

Les stages internes ne sont rien d'autre qu'une extension des journées de traduction prévues pour la première année. L'extension est une extension en durée, puisque l'on passerait de la journée à la semaine, et une extension dans la matière, puisque ces stages internes pourraient être envisagés en rapport avec les enseignements de rédaction, de révision ou de terminologie. La notion de stage interne appelle une redistribution du calendrier des études et une étroite collaboration entre enseignants responsables de divers cours. Le stage interne présenterait les caractéristiques suivantes, l'exemple de référence étant celui du stage de traduction :

- premier jour : remise aux participants d'un texte de 4000 mots environ ou d'une série homogène de textes dont l'ensemble compterait le même nombre de

5. La révision externe en première année d'école de traduction présenterait l'avantage de poser le problème des évaluations sous un jour « réaliste $\$$. Nul ne discute les commentaires et appréciations d'une personnalité «extérieure 》 investie de l'autorité que lui confère la pratique professionnelle et que pourrait lui conférer sa «neutralité » pédagogique. Cette même révision externe présenterait l'avantage supplémentaire de donner aux étudiants une idée très précise de ce que représente la révision et de ce qu'elle implique $-\mathrm{y}$ compris, pour certains, du chemin à parcourir et de l'effort à accomplir, 
mots, dépouillement du texte par les étudiants, relevé des difficultés présumées, début de la recherche documentaire - avec ou sans l'aide d'un enseignant apportant des directives mais pas de solutions ;

- second jour : documentation et recherches terminologiques. Ces activités se dérouleraient, de préférence, en présence du responsable des cours de documentation et/ou de terminologie qui peut alors vérifier la portée et la méthode des recherches et dispenser des conseils touchant à l'organisation du travail ;

- troisième et quatrième jours : pré-traduction et traduction du texte : ces deux exercices se dérouleraient en présence de l'enseignant responsable du cours de traduction mais aussi, le cas échéant, en présence des professeurs de terminologie et documentation qui pourraient alors juger de l'efficacité immédiate des recherches entreprises les jours précédents ;

- cinquième jour : révision individuelle de la traduction, mise au point de la version définitive, vérifications et dactylographie.

L'ampleur du travail fait que, cette fois; la présence de plusieurs enseignants - en même temps ou à tour de rôle - devient nécessaire. Dans cette hypothèse, les avantages offerts par le stage interne sont les mêmes que ceux offerts par les journées de traduction. Leur portée est cependant plus grande dans la mesure où les textes traités sont plus longs et dans la mesure aussi où la pression s'exerce plus longuement.

L'extension du stage interne à d'autres types d'exercices ne nécessiterait qu'un aménagement du calendrier. Il serait possible de prévoir, pour un groupe d'étudiants donné, des séries de stages consécutifs touchant l'ensemble des matières de cours.

Il pourrait ainsi y avoir :

- un stage de terminologie. Ce stage pourrait se construire autour d'une recherche thématique allant de la recherche des documents à leur exploitation puis à la mise au point d'un répertoire ou mini-glossaire. Les travaux pourraient être organisés en travaux collectifs par découpage du thème en sous-thèmes. Le stage de terminologie pourrait, le cas échéant, comporter des phases de recherche ponctuelle au cours desquelles les étudiants devraient répondre aux demandes faites par un utilisateur de terminologie. Le stage interne de terminologie pourrait également déboucher sur des activités externes (consultations auprès de sources extérieures au cadre universitaire ou au milieu professionnel);

- un stage de rédaction;

- un stage de révision.

D'autre part, il serait possible de démultiplier les stages internes de traduction en prévoyant un stage de traduction générale, un stage de traduction spécialisée, un stage de traduction technique. Il n'est certes pas nécessaire de jouir d'une imagination débordante pour prévoir une grille d'activités concernant ces divers stages. Nul doute que chaque responsable d'un cours saurait fort bien comment occuper les journées des étudiants. 
Phase trois : les stages externes - troisième et dernière année de formation universitaire.

Certains employeurs - au nombre desquels figure en bonne place le Bureau des traductions et certaines universités — dont l'Université Laval — ont déjà mis en place une structure de stages « externes ». L'opinion émise par les stagiaires est sans équivoque et ce genre de stage externe est sans aucun doute la transition nécessaire entre le milieu universitaire et le milieu professionnel. Il est indiscutable que ces stages ne sont possibles que si toutes les écoles de traduction et tous les employeurs s'accordent tout d'abord à en reconnaître la nécessité et acceptent ensuite de collaborer afin de les organiser. Tout porte à croire que les obstacles ne sont pas insurmontables. Mais la bonne volonté des enseignants restera lettre morte tant que tous les employeurs n'apporteront pas leur appui inconditionnel. Si tous les étudiants de toutes les écoles de traduction avaient la possibilité de suivre un stage pratique d'une durée minimale d'un mois dans une section ou un bureau de traduction, ils ne seraient pas pour autant «opérationnels » mais ils seraient certainement "dégrossis 》 et moins tendus à la perspective d'un départ dans la vie professionnelle.

\section{LES CONTRAINTES D'ORGANISATION DES STAGES}

L'organisation des stages étant conditionnée par les possibilités offertes par le milieu non universitaire, nous nous contenterons d'analyser les contraintes posées par les stages internes et de proposer un schéma d'aménagement de l'année universitaire acceptant cette «greffe».

La première hypothèse concerne l'organisation d'un stage interne d'une durée d'un mois et portant sur une série de quatre stages d'une semaine chacun qui seraient : un stage en traduction générale, un stage en traduction technique ou spécialisée, un stage en rédaction et révision et un stage en terminologie.

L'unité-stage comprendrait quatre semaines d'exercices plus deux semaines consacrées aux bilans. Il faudrait donc, dans le calendrier universitaire, dégager une période de six semaines franches. Pendant ces six semaines, les étudiants seraient en stage en ce sens qu'ils ne suivraient aucun cours. L'idéal serait que ce stage se situe au cours des six dernières semaines de l'année universitaire. L'arrêt des cours offrirait un double avantage : tout d'abord, les enseignants seraient en mesure d'assister et/on de participer au stage ou, plus précisément, aux stages; en second lieu, les enseignants responsables de l'un ou l'autre stage disposeraient de temps pour corriger les travaux (si le stage qu'ils organisent se situe en tête de série) ou pour préparer leur stage. Il est entendu que l'enseignant dont le stage intervient en fin de série dispose de moins de temps pour la correction des travaux mais le bilan de son stage interviendrait également en dernier lieu, ce qui lui laisserait une semaine et demie pour assurer les corrections (puisque les bilans occupent deux semaines iet concernent quatre stages différents). Le seul problème qui subsisterait est celui des cours pour lesquels des stages ne sont pas prévus. La solution est double : ou bien l'horaire de ces cours se trouve renforcé pendant la période précédant le début des stages, ou bien chacun des stages est 
raccourci d'une journée. Dans l'hypothèse où chacun des stages est amputé d'une journée, cette journée libérée serait réservée aux cours sans stage. Dans ces circonstances, ou bien la portée du stage se trouve réduite en conséquence, ou bien les étudiants doivent consacrer à la mise au point finale et à la dactylographie des travaux une partie de la fin de semaine.

Le schéma correspondant à l'organisation d'une série de stages en fin d'année universitaire, sans préjudice des cours sans stage, serait le suivant :

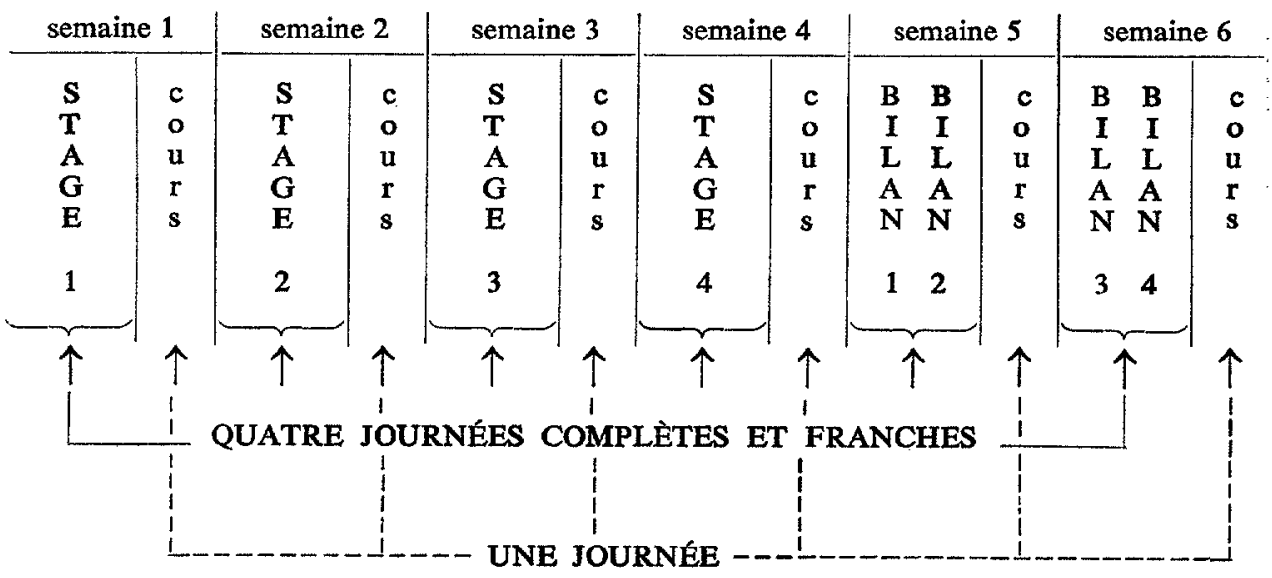

La seconde hypothèse concerne l'organisation éventuelle de deux séries de stages suivies des deux séries de bilans. Cette seconde hypothèse supposerait une réorganisation en profondeur de l'année universitaire. L'idéal serait que l'année soit découpée en quatre tranches de six semaines :

- première tranche de six semaines : grille normale de cours;

- seconde tranche de six semaines : stages suivis de bilans;

- troisième tranche de six semaines : grille normale de cours ;

— quatrième tranche de six semaines : stages suivis de bilans.

L'avantage de la double série de stages réside dans la possibilité de prévoir un premier stage "dirigé » et un second stage «libre». La différnence résiderait dans l'encadrement relativement rigide du premier stage et le non-encadrement du second.

En d'autres termes, au cours du premier stage, le responsable peut imposer une progression rigide mais, au cours du second, il laisse pleine et entière liberté d'organisation aux étudiants. L'autre avantage de la double série de stages, outre le fait que le premier permet d'essuyer les plâtres afin de rentabiliser le second, naît de l'augmentation sensible du nombre de travaux pratiques. L'inconvénient de la double série est, naturellement, la multiplication par deux de l'inconvénient de la première, à savoir, le problème des cours sans stage.

Il existerait deux façons de pallier cet inconvénient majeur. Tout d'abord, il serait possible de doubler, pendant les périodes sans stage, les horaires des cours 
sans stages. Ceci fonctionnerait à merveille pour les cours dont le contenu peut se découper en tranches et, à plus forte raison, pour les cours sanctionnés par un double examen. Peut-être ne serait-il pas mauvais de laisser parfois se décanter les connaissances? Il est cependant probable que cette solution constitue, aux yeux de nombreux enseignants un remède pire que le mal. Il faudrait donc revenir à l'option retenue dans l'hypothèse de la série isolée de stages. Le schéma général de l'année serait alors le suivant (schéma valant pour un semestre dont le second serait la réplique exacte) :

\begin{tabular}{|c|c|c|}
\hline grille normale des cours & $\begin{array}{l}\text { grille de série d } \\
\text { (reprenant le prem }\end{array}$ & $\begin{array}{l}\text { stages et bilans } \\
\text { er schéma ci-dessus) }\end{array}$ \\
\hline 6 semaines & $\begin{array}{l}4 \text { semaines } \\
\text { stages }\end{array}$ & $\begin{array}{c}\text { deux semaines } \\
\text { bilans }\end{array}$ \\
\hline
\end{tabular}

Il resterait sans doute à régler le problème des notes sans lesquelles un programme semble ne pas pouvoir fonctionner. Ce dernier est d'autant plus facile à régler qu'il ne se pose même pas : peut-on rêver travaux pratiques plus pratiques que ceux effectués en stage - surtout si l'on a pris la peine d'évaluer en cours de stage les aptitudes générales du stagiaire ? Ne peut-on pas, en fin de stage, faire subir aux participants une épreuve en temps limité portant sur un exercice bref ayant un rapport direct avec le thème abordé ? Les stages ne doivent pas être considérés comme une activité annexe mais bien comme l'activité principale autour de laquelle s'organisent les cours. Ils permettent en effet, pour l'étudiant, une application des principes et méthodes exposés en cours et, pour l'enseignant, une meilleure définition des lacunes et carences réelles ainsi que des solutions et remèdes.

\section{BILAN}

La mise en place de journées de traduction, stages internes et stages externes oblige à :

— une redéfinition partielle ou totale du profil de formation des étudiants en traduction ;

— une refonte des emplois du temps et de la distribution des cours;

- un glissement vers une orientation plus nettement professionnelle;

- une modification des options pédagogiques avec substitution, à l'analyse extérieure des résultats de travaux, d'une analyse directe des démarches et stratégies des étudiants ;

- une organisation des études en traduction selon une double grille verticale tet horizontale : coordination par année autour des séries de stages appelant une nécessaire collaboration entre enseignants ; coordination d'année en année dans le 
passage de la « journée » à la « semaine», puis de l'université (stage interne) à la « réalité 》(stage externe) ;

- une collaboration étroite entre enseignants et employeurs dans l'organisation des stages externes.

La progression en trois phases aurait pour effet de déterminer une ossature pyramidale dont le sommet serait la porte ouvrant sur la vie professionnelle. Il est sans conteste souhaitable que des écoles, que la lucidité oblige à décrire comme des écoles à vocation professionnelle, ne soient pas déguisées en départements universitaires ou, du moins, ne soient pas tenues de fonctionner sur le modèle d'institutions à vocation non professionnelle. Ceci ne signifie nullement que les écoles puissent rêver de livrer à tous les employeurs un «produit fini 》 qui corresponde en tout point à leurs exigences. La diversité des emplois ferait de cet objectif une vue d'utopiste. Il n'est pas exclu non plus que les écoles de traduction aient, de par la formation «gratuite » dispensée, un rôle important et fort utile à jouer dans la revitalisation ou la réévaluation de la pratique professionnelle - n'en déplaise à leurs détracteurs. Quoi qu'il en soit, nous pensons, avec bien d'autres, que les écoles de traduction pourraient (devraient) assurer des programmes para-professionnels dans lesquels se fondraient progressivement la formation générale du traducteur et la pré-formation professionnelle en réponse aux exigences des employeurs. Tout programme (para)-professionnel s'articule autour de stages. Il existe déjà des stages externes. Si ceux-là ont pu se mettre en place c'est qu'il y a eu volonté de mise en place de part et d'autre. S'ils ont pu être organisés, pourquoi serait-il impossible d'organiser des stages internes ? Et si ces stages internes peuvent être organisés, pourquoi pas, à plus forte raison, les journées de traduction ${ }^{6}$ ?

6. Nous tenons à remercier M. Brian Harris, directeur de l'École de traducteurs et d'interprètes de l'Université d'Ottawa, ainsi que l'ensemble du corps professoral de cette même école pour les encouragements prodigués et l'aide précieuse apportée à l'occasion de ces journées de traduction - en particulier dans l'aménagement de l'emploi du temps. 